\title{
A bunch of time integrators for quantum/classical molecular dynamics
}

\author{
Marlis Hochbruck and Christian Lubich \\ Mathematisches Institut, Universität Tübingen, Auf der Morgenstelle 10, \\ D-72076 Tübingen, Germany. \\ E-mail: marlis@@na.uni-tuebingen.de, lubich@@na.uni-tuebingen.de
}

\begin{abstract}
We present novel time integration schemes for Newtonian dynamics whose fastest oscillations are nearly harmonic, for constrained Newtonian dynamics including the Car-Parrinello equations of $a b$ initio molecular dynamics, and for mixed quantum-classical molecular dynamics. The methods attain favorable properties by using matrix-function vector products which are computed via Lanczos' method. This permits to take longer time steps than in standard integrators.
\end{abstract}

\section{Introduction}

In this paper we present a number of time integrators for various problems ranging from classical to quantum molecular dynamics. These integrators share some common features: they are new, they are second-order accurate and time-reversible, they improve substantially over standard schemes in welldefined model situations - and none of them has been tested on real applications at the time of this writing. This last feature will hopefully change in the near future [20].

On a more technical level, a further common feature of all the schemes proposed here is that they require the computation of the product of an analytic function of a symmetric matrix with a vector. Integration schemes employing matrix functions apparently have not hitherto been used in practice, except in a few special cases where direct diagonalization is possible. However, since the mid-eighties, starting with a paper by Park and Light [21] on quantum propagators, Lanczos' method has been put to good use in approximating matrix-function vector products. More recently, the excellent convergence properties of this approach have been clarified in $[7,12]$. This motivated the development of the general-purpose ODE integrator exp4 in [15]. Employing matrix functions in an integrator enables us to obtain favorable properties, such as exact integration of linear differential systems with constant coefficients. This is very advantageous for problems where the fastest oscillations are nearly harmonic. Matrix functions add a welcome element

Computational Molecular Dynamics: Challenges, Methods, Ideas, P. Deuflhard, J. Hermans, B. Leimkuhler, A. Mark, S. Reich, and R.D. Skeel, eds., Springer Lecture Notes in Computational Science and Engineering, Vol. 4, pages 121-132, 1998. 
to the construction of integration schemes. The freedom thus gained can be used for designing new integrators tailored to specific applications, as we try to demonstrate with the methods in this paper. A theoretical error analysis of these schemes is given in [13,14]. We hope that the proposed integrators, or closely related ones, will be found useful in molecular dynamics.

\section{Newtonian equations of motion}

In this section we consider the classical equations of motion of particles in cases where the highest-frequency oscillations are nearly harmonic: The positions $\mathbf{y}(t)=\left\{y_{i}(t)\right\}$ evolve according to the second-order system of differential equations

$$
\mathbf{M} \ddot{\mathbf{y}}=-\mathbf{A y}+\mathbf{f}(\mathbf{y}) .
$$

Here, $\mathbf{M}$ is a constant, symmetric positive definite mass matrix. We assume without loss of generality that $\mathbf{M}$ is simply the identity matrix $\mathbf{I}$. Otherwise, this is achieved by the familiar transformation

$$
\mathbf{y} \mapsto \mathbf{M}^{1 / 2} \mathbf{y}, \quad \mathbf{A} \mapsto \mathbf{M}^{-1 / 2} \mathbf{A M}^{-1 / 2}, \quad \mathbf{f}(\mathbf{y}) \mapsto \mathbf{M}^{-1 / 2} \mathbf{f}\left(\mathbf{M}^{-1 / 2} \mathbf{y}\right)
$$

We assume that $\mathbf{A}$ is a symmetric and positive semi-definite matrix. The case of interest is when the largest eigenvalue of $\mathbf{A}$ is significantly larger than the norm of the derivative of the nonlinear force $\mathbf{f}$. $\mathbf{A}$ may be a constant matrix, or else $\mathbf{A}=\mathbf{A}(\mathbf{y})$ is assumed to be slowly changing along solution trajectories, in which case $\mathbf{A}$ will be evaluated at the current averaged position in the numerical schemes below. In the standard Verlet scheme, which yields approximations $\mathbf{y}^{n}$ to $\mathbf{y}(n \Delta t)$ via

$$
\mathbf{y}^{n+1}-2 \mathbf{y}^{n}+\mathbf{y}^{n-1}=\Delta t^{2}\left(-\mathbf{A} \mathbf{y}^{n}+\mathbf{f}\left(\mathbf{y}^{n}\right)\right),
$$

the time step $\Delta t$ is then limited by the inverse of the largest eigenvalue of $\mathbf{A}$. We are aiming at methods whose step size is restricted only by the nonlinearity $\mathbf{f}$. To motivate the derivation of such a scheme, we start from the linear equation with constant force vector

$$
\ddot{\mathbf{y}}=-\mathbf{A y}+\mathbf{f} \quad(\mathbf{A}, \mathbf{f} \text { constant })
$$

whose exact solution satisfies

$$
\mathbf{y}(t+\Delta t)-2 \mathbf{y}(t)+\mathbf{y}(t-\Delta t)=\Delta t^{2} \sigma\left(\Delta t^{2} \mathbf{A}\right)(-\mathbf{A y}(t)+\mathbf{f})
$$

with the complex function

$$
\sigma(z)=\left(\frac{\sin (\sqrt{z} / 2)}{\sqrt{z} / 2}\right)^{2} .
$$


Approximating the nonlinear force $\mathbf{f}(\mathbf{y})$ over a time step by a suitable constant vector leads to a scheme whose origins for scalar equations can be traced back to [10]:

$$
\mathbf{y}^{n+1}-2 \mathbf{y}^{n}+\mathbf{y}^{n-1}=\Delta t^{2} \sigma\left(\Delta t^{2} \mathbf{A}\right)\left(-\mathbf{A} \mathbf{y}^{n}+\mathbf{f}^{n}\right)
$$

with

$$
\mathbf{f}^{n}=\mathbf{f}\left(\overline{\mathbf{y}}^{n}\right) .
$$

Here, the obvious choice would be $\overline{\mathbf{y}}^{n}=\mathbf{y}^{n}$. However, as was first proposed in [8] for a different method described at the end of this section, it turns out to be favorable to take an averaged position value

$$
\overline{\mathbf{y}}^{n}=\phi\left(\Delta t^{2} \mathbf{A}\right) \mathbf{y}^{n}
$$

with a suitably chosen filter function $\phi(z)$, or eventually a translated version of (6), with some reference position $\mathbf{y}^{*}$,

$$
\overline{\mathbf{y}}^{n}=\mathbf{y}^{*}+\phi\left(\Delta t^{2} \mathbf{A}\right)\left(\mathbf{y}^{n}-\mathbf{y}^{*}\right) .
$$

The choice of $\phi$ strongly influences the mixing of frequencies by the nonlinearity. Two possible choices are

$$
\phi(z)=\phi_{0}(z)=\frac{\sin \sqrt{z}}{\sqrt{z}}
$$

which is a filter function suggested in [8], or preferably

$$
\phi(z)=\phi_{1}(z)=\left(1+\frac{1}{6}(1-\cos \sqrt{z})\right) \phi_{0}(z) .
$$

Of course, to make the scheme (4) practical, we must be able to compute the products of matrix functions $\sigma\left(\Delta t^{2} \mathbf{A}\right)$ and $\phi\left(\Delta t^{2} \mathbf{A}\right)$ with vectors efficiently. This will be discussed in Section 5 .

From the derivation of the method (4) it is obvious that the scheme is exact for constant-coefficient linear problems (3). Like the Verlet scheme, it is also time-reversible. For the special case $\mathbf{A}=\mathbf{0}$ it reduces to the Verlet scheme. It is shown in [13] that the method has an $O\left(\Delta t^{2}\right)$ error bound over finite time intervals for systems with bounded energy. In contrast to the Verlet scheme, this error bound is independent of the size of the eigenvalues $\lambda_{k}$ of $\mathbf{A}$.

It turns out that the error is essentially determined by a two-dimensional scalar function $\epsilon(x, y)$ evaluated at $x=\omega_{k} \Delta t, y=\omega_{l} \Delta t$, where $\omega_{k}=\sqrt{\lambda_{k}}$ are the frequencies of the linear system (3). This error function depends strongly on the choice of $\phi$. The most obvious choice $\phi \equiv 1$ suffers from resonances, which express themselves in singularities of $\epsilon$ at arguments that are integer multiples of $\pi$. This leads to a loss of accuracy in resonance situations. The same is true for the choice $\phi=\sigma$. For $\phi$ given by (7) and (8) no such problems occur. The choice (8) has the advantage that it drastically reduces the error 
function $|\epsilon(x, y)|$ for small $x, y$ and therefore gives better accuracy. See [13] for more details on the error analysis.

A widely used variant of the Verlet scheme is its velocity version:

$$
\begin{aligned}
\mathbf{v}^{n+1 / 2} & =\mathbf{v}^{n}+\frac{\Delta t}{2}\left(-\mathbf{A} \mathbf{y}^{n}+\mathbf{f}^{n}\right) \\
\mathbf{y}^{n+1} & =\mathbf{y}^{n}+\Delta t \mathbf{v}^{n+1 / 2} \\
\mathbf{v}^{n+1} & =\mathbf{v}^{n+1 / 2}+\frac{\Delta t}{2}\left(-\mathbf{A} \mathbf{y}^{n+1}+\mathbf{f}^{n+1}\right)
\end{aligned}
$$

with $\mathbf{f}^{n}=\mathbf{f}\left(\mathbf{y}^{n}\right)$. Similarly, also the scheme (4) admits a velocity version, which this time works with averaged velocities

$$
\mathbf{v}^{n} \approx \phi_{0}\left(\Delta t^{2} \mathbf{A}\right) \dot{\mathbf{y}}(n \Delta t) \approx \frac{1}{2 \Delta t} \int_{-\Delta t}^{\Delta t} \dot{\mathbf{y}}(n \Delta t+\tau) d \tau
$$

This one-step version of (4) reads (for $\mathbf{f}^{n}$ of (5) with $\overline{\mathbf{y}}^{n}$ of (6))

$$
\begin{aligned}
\mathbf{v}^{n+1 / 2} & =\mathbf{v}^{n}+\frac{\Delta t}{2} \sigma\left(\Delta t^{2} \mathbf{A}\right)\left(-\mathbf{A} \mathbf{y}^{n}+\mathbf{f}^{n}\right) \\
\mathbf{y}^{n+1} & =\mathbf{y}^{n}+\Delta t \mathbf{v}^{n+1 / 2} \\
\mathbf{v}^{n+1} & =\mathbf{v}^{n+1 / 2}+\frac{\Delta t}{2} \sigma\left(\Delta t^{2} \mathbf{A}\right)\left(-\mathbf{A} \mathbf{y}^{n+1}+\mathbf{f}^{n+1}\right) .
\end{aligned}
$$

A different "long-time-step method" was previously proposed by GarcíaArchilla, Sanz-Serna, and Skeel [8]. Their mollified impulse method, which is based on the concept of operator splitting and also reduces to the Verlet scheme for $\mathbf{A}=\mathbf{0}$ and admits second-order error estimates independently of the frequencies of $\mathbf{A}$, reads as follows when applied to (1):

$$
\begin{aligned}
\mathbf{v}_{+}^{n} & =\mathbf{v}^{n}+\frac{\Delta t}{2} \phi\left(\Delta t^{2} \mathbf{A}\right) \mathbf{f}^{n} \\
\left(\begin{array}{c}
\mathbf{y}^{n+1} \\
\mathbf{v}_{-}^{n+1}
\end{array}\right) & =\exp \left(\Delta t\left[\begin{array}{cc}
\mathbf{0} & \mathbf{I} \\
-\mathbf{A} & \mathbf{0}
\end{array}\right]\right)\left(\begin{array}{l}
\mathbf{y}^{n} \\
\mathbf{v}_{+}^{n}
\end{array}\right) \\
\mathbf{v}^{n+1} & =\mathbf{v}_{-}^{n+1}+\frac{\Delta t}{2} \phi\left(\Delta t^{2} \mathbf{A}\right) \mathbf{f}^{n+1}
\end{aligned}
$$

where $\mathbf{f}^{n}$ is again given by (5) and (6). Eliminating the (non-averaged) velocities, this scheme can be shown to become

$$
\mathbf{y}^{n+1}-2 \mathbf{y}^{n}+\mathbf{y}^{n-1}=\Delta t^{2} \sigma\left(\Delta t^{2} \mathbf{A}\right)\left(-\mathbf{A} \mathbf{y}^{n}+\mathbf{f}^{n}\right)+\Delta t^{2} \delta\left(\Delta t^{2} \mathbf{A}\right) \mathbf{f}^{n}
$$

where $\delta(z)=\phi(z) \phi_{0}(z)-\sigma(z)$. A comparison of theoretical properties of (10) and (11) is given in [13]. 


\section{Car-Parrinello equations of ab initio molecular dynamics, constrained Newtonian dynamics}

In the Car-Parrinello method [6] (and see, e.g., [24,25,16,4]), the adiabatic time-dependent Born-Oppenheimer model is approximated by a fictitious Newtonian dynamics in which the electrons, represented by a set of wave functions $\left\{\left|\psi_{j}\right\rangle\right\}$, follow the motion of the ions, represented by a set of positions $\left\{\mathbf{R}_{J}\right\}$. The model involves a small fictitious-mass parameter $\mu$ which keeps the fictitious kinetic energy of the electrons small compared to the kinetic energy of the ions. The dynamics is determined by the Kohn-Sham energy functional $E\left(\left\{\mathbf{R}_{J}\right\},\left\{\left|\psi_{j}\right\rangle\right\}\right)$, and by the orthonormality constraint for the orbitals. The Euler-Lagrange equations of motion then read, with Lagrange multipliers $\Lambda_{j k}$,

$$
\begin{aligned}
M_{J} \ddot{\mathbf{R}}_{J} & =-\frac{\partial E}{\partial \mathbf{R}_{J}} \\
\mu\left|\ddot{\psi}_{j}\right\rangle & =-\frac{\partial E}{\partial\left|\psi_{j}\right\rangle}+\sum_{k} \Lambda_{j k}\left|\psi_{k}\right\rangle \\
\left\langle\psi_{j} \mid \psi_{k}\right\rangle & =\delta_{j k} .
\end{aligned}
$$

After spatial (spectral) discretization, this becomes a large finite-dimensional system in the evolution variables $\mathbf{y}=\left[\left\{\mathbf{R}_{J}\right\},\left\{\boldsymbol{\psi}_{j}\right\}\right]$ of the form

$$
\begin{aligned}
\ddot{\mathbf{y}} & =-\mathbf{A y}+\mathbf{f}(\mathbf{y})+\mathbf{G}^{T}(\mathbf{y}) \boldsymbol{\lambda} \\
\mathbf{g}(\mathbf{y}) & =\mathbf{0}
\end{aligned}
$$

where $\mathbf{G}=\partial \mathbf{g} / \partial \mathbf{y}$. The splitting of the forces might be such that $\mathbf{A}$ represents the discretized Laplacian contained in the Kohn-Sham Hamiltonian, or, e.g., $\mathbf{A}=\mathbf{A}(\mathbf{y})$ contains in addition the Jacobian of the local interaction forces. The choice of the most effective splitting may depend on physical insight into the specific problem at hand, and quite probably on extensive numerical experiments.

The standard numerical integrators for the constrained system (12) are the SHAKE scheme [23], which extends the Verlet method (2),

$$
\begin{aligned}
\mathbf{y}^{n+1}-2 \mathbf{y}^{n}+\mathbf{y}^{n-1} & =\Delta t^{2}\left(-\mathbf{A} \mathbf{y}^{n}+\mathbf{f}\left(\mathbf{y}^{n}\right)+\mathbf{G}^{T}\left(\mathbf{y}^{n}\right) \boldsymbol{\lambda}^{n}\right) \\
\mathbf{g}\left(\mathbf{y}^{n+1}\right) & =\mathbf{0}
\end{aligned}
$$

and the RATTLE scheme [1], which extends the velocity Verlet method (9). There, one first solves for $\mathbf{y}^{n+1}$ in

$$
\begin{aligned}
\mathbf{v}^{n+1 / 2} & =\mathbf{v}^{n}+\frac{\Delta t}{2}\left(-\mathbf{A} \mathbf{y}^{n}+\mathbf{f}\left(\mathbf{y}^{n}\right)+\mathbf{G}^{T}\left(\mathbf{y}^{n}\right) \boldsymbol{\lambda}^{n}\right) \\
\mathbf{y}^{n+1} & =\mathbf{y}^{n}+\Delta t \mathbf{v}^{n+1 / 2} \\
\mathbf{g}\left(\mathbf{y}^{n+1}\right) & =\mathbf{0}
\end{aligned}
$$


and subsequently for $\mathbf{v}^{n+1}$ in

$$
\begin{aligned}
& \mathbf{v}^{n+1}=\mathbf{v}^{n+1 / 2}+\frac{\Delta t}{2}\left(-\mathbf{A} \mathbf{y}^{n+1}+\mathbf{f}\left(\mathbf{y}^{n+1}\right)+\mathbf{G}^{T}\left(\mathbf{y}^{n+1}\right) \widehat{\boldsymbol{\lambda}}^{n+1}\right) \\
& \mathbf{G}\left(\mathbf{y}^{n+1}\right) \mathbf{v}^{n+1}=\mathbf{0} .
\end{aligned}
$$

In the same ways, the scheme (4) extends to

$$
\begin{aligned}
\mathbf{y}^{n+1}-2 \mathbf{y}^{n}+\mathbf{y}^{n-1} & =\Delta t^{2} \sigma\left(\Delta t^{2} \mathbf{A}\right)\left(-\mathbf{A} \mathbf{y}^{n}+\mathbf{f}\left(\overline{\mathbf{y}}^{n}\right)+\mathbf{G}^{T}\left(\overline{\mathbf{y}}^{n}\right) \boldsymbol{\lambda}^{n}\right) \\
\mathbf{g}\left(\mathbf{y}^{n+1}\right) & =\mathbf{0},
\end{aligned}
$$

and its averaged-velocity version (10) extends to

$$
\begin{aligned}
\mathbf{v}^{n+1 / 2} & =\mathbf{v}^{n}+\frac{\Delta t}{2} \sigma\left(\Delta t^{2} \mathbf{A}\right)\left(-\mathbf{A} \mathbf{y}^{n}+\mathbf{f}\left(\overline{\mathbf{y}}^{n}\right)+\mathbf{G}^{T}\left(\overline{\mathbf{y}}^{n}\right) \boldsymbol{\lambda}^{n}\right) \\
\mathbf{y}^{n+1} & =\mathbf{y}^{n}+\Delta t \mathbf{v}^{n+1 / 2} \\
\mathbf{g}\left(\mathbf{y}^{n+1}\right) & =\mathbf{0}
\end{aligned}
$$

in the first half-step, and

$$
\begin{aligned}
& \mathbf{v}^{n+1}=\mathbf{v}^{n+1 / 2}+\frac{\Delta t}{2} \sigma\left(\Delta t^{2} \mathbf{A}\right)\left(-\mathbf{A} \mathbf{y}^{n+1}+\mathbf{f}\left(\overline{\mathbf{y}}^{n+1}\right)+\mathbf{G}^{T}\left(\overline{\mathbf{y}}^{n+1}\right) \widehat{\boldsymbol{\lambda}}^{n+1}\right) \\
& \mathbf{G}\left(\overline{\mathbf{y}}^{n+1}\right) \mathbf{v}^{n+1}=\mathbf{0}
\end{aligned}
$$

in the second half-step. Here $\overline{\mathbf{y}}^{n}$ is again defined as in (6).

Both methods are time-reversible. For $\mathbf{A}=\mathbf{0}$, they reduce to SHAKE and RATTLE. In contrast to SHAKE and RATTLE, the time step is not restricted by the largest eigenvalue of $\mathbf{A}$.

\section{Quantum-classical molecular dynamics}

In the mixed quantum-classical molecular dynamics (QCMD) model (see $[11,9,2,3,5]$ and references therein), most atoms are described by classical mechanics, but an important small portion of the system by quantum mechanics. The full quantum system is first separated via a tensor product ansatz. The evolution of each part is then modeled either classically or quantally. This leads to a coupled system of Newtonian and Schrödinger equations.

For ease of presentation only, we here consider the case of two particles having spatial coordinates $\mathbf{x}$ and $\mathbf{y}$, and masses $m$ and $M$, with $m \ll M$. With the interaction potential $V(\mathbf{x}, \mathbf{y})$, the quantum Hamiltonian $H$ is given by

$$
H(\mathbf{y})=-\frac{\hbar^{2}}{2 m} \Delta_{\mathbf{x}}+V(\mathbf{x}, \mathbf{y}) .
$$

The equations of motion of the QCMD model read

$$
\begin{aligned}
M \ddot{\mathbf{y}} & =-\left\langle\psi\left|H^{\prime}(\mathbf{y})\right| \psi\right\rangle \\
i \hbar|\dot{\psi}\rangle & =H(\mathbf{y})|\psi\rangle
\end{aligned}
$$


where $H^{\prime}=\nabla_{\mathbf{y}} H=\nabla_{\mathbf{y}} V$. After spatial discretization in $\mathbf{x}$, we obtain a large finite-dimensional system

$$
\begin{aligned}
M \ddot{\mathbf{y}} & =-\boldsymbol{\psi}^{*} \mathbf{H}^{\prime}(\mathbf{y}) \boldsymbol{\psi} \\
i \hbar \dot{\boldsymbol{\psi}} & =\mathbf{H}(\mathbf{y}) \boldsymbol{\psi}
\end{aligned}
$$

with the matrix $\mathbf{H}(\mathbf{y})=\mathbf{T}+\mathbf{V}(\mathbf{y})$, where both $\mathbf{T}$ and $\mathbf{V}(\mathbf{y})$ are real and symmetric, and $\mathbf{T}$ is positive semi-definite. $\mathbf{T}$ is a constant matrix (the discretized Laplacian) of which we assume no bounds. On the other hand, $\mathbf{V}(\mathbf{y})$ and its first two derivatives are assumed to be moderately bounded. Typically, $\mathbf{T}$ is transformed to diagonal form by discrete Fourier transforms, and $\mathbf{V}(\mathbf{y})$ is a diagonal matrix.

A quite successful integrator for (16), which is based on the idea of operator splitting, is the PICKABACK scheme of Nettesheim et al. [19]. With the shorthand notation

$$
\mathrm{i}=i / \hbar
$$

their scheme reads

$$
\begin{aligned}
\mathbf{y}^{n+1 / 2} & =\mathbf{y}^{n}+\frac{\Delta t}{2} \mathbf{v}^{n} \\
\boldsymbol{\psi}^{n+1 / 2} & =\exp \left(-\mathrm{i} \frac{\Delta t}{2} \mathbf{T}\right) \boldsymbol{\psi}^{n} \\
\mathbf{v}^{n+1} & =\mathbf{v}^{n}-\frac{\Delta t}{M}\left(\boldsymbol{\psi}^{n+1 / 2}\right)^{*} \mathbf{H}^{\prime}\left(\mathbf{y}^{n+1 / 2}\right) \boldsymbol{\psi}^{n+1 / 2} \\
\boldsymbol{\psi}^{n+1} & =\exp \left(-\mathrm{i} \frac{\Delta t}{2} \mathbf{T}\right) \exp \left(-\mathrm{i} \Delta t \mathbf{V}\left(\mathbf{y}^{n+1 / 2}\right)\right) \boldsymbol{\psi}^{n+1 / 2} \\
\mathbf{y}^{n+1} & =\mathbf{y}^{n+1 / 2}+\frac{\Delta t}{2} \mathbf{v}^{n+1} .
\end{aligned}
$$

This scheme requires the exponential only of matrices that are diagonal or transformed to diagonal form by fast Fourier transforms. Unfortunately, this matrix splitting leads to time step restrictions of the order of the inverse of the largest eigenvalue of $\mathbf{T} / \hbar$. A simple, Verlet-like scheme that uses no matrix splitting, is the following:

$$
\begin{aligned}
\mathbf{y}^{n+1}-2 \mathbf{y}^{n}+\mathbf{y}^{n-1} & =-\frac{\Delta t^{2}}{M}\left(\boldsymbol{\psi}^{n}\right)^{*} \mathbf{H}^{\prime}\left(\mathbf{y}^{n}\right) \boldsymbol{\psi}^{n} \\
\boldsymbol{\psi}^{n} & =\exp \left(-\mathrm{i} \frac{\Delta t}{2} \mathbf{H}^{n}\right) \boldsymbol{\psi}^{n-1 / 2} \\
\boldsymbol{\psi}^{n+1 / 2} & =\exp \left(-\mathrm{i} \frac{\Delta t}{2} \mathbf{H}^{n}\right) \boldsymbol{\psi}^{n}=\exp \left(-\mathrm{i} \Delta t \mathbf{H}^{n}\right) \boldsymbol{\psi}^{n-1 / 2}(20)
\end{aligned}
$$

with $\mathbf{H}^{n}=\mathbf{H}\left(\mathbf{y}^{n}\right)$. We note that here (and in the schemes to follow) the $\mathbf{y}$-recursion could be rewritten in the velocity form (9). The action of the exponential is now approximated by the Lanczos method described in Section 5, with a Krylov subspace corresponding to the matrix $\mathbf{H}^{n}$ and the 
vector $\boldsymbol{\psi}^{n-1 / 2}$. Like (17), the scheme (18)-(20) is time-reversible, is unitary in the quantum part, evaluates the Hamiltonian and its gradient at the same position, and has formal order of accuracy 2. However, second-order error bounds independently of the highest frequencies can be obtained only under unreasonable smoothness assumptions about the solution.

In the following we devise, following [14], an efficiently implementable scheme which leads to favorable error bounds independently of the highest frequencies under the mere assumption that the system has bounded energy. The scheme will be time-reversible, and robust in the singular limit of the mass ratio $m / M$ tending to 0 .

We first deal with the $\mathbf{y}$-equation. We start from the identity

$$
\mathbf{y}(t+\Delta t)-2 \mathbf{y}(t)+\mathbf{y}(t-\Delta t)=\int_{0}^{\Delta t}(\Delta t-\tau)(\ddot{\mathbf{y}}(t+\tau)+\ddot{\mathbf{y}}(t-\tau)) d \tau
$$

which leads us to replace the pointwise force evaluation of (18) by

$$
\begin{aligned}
\mathbf{y}^{n+1}-2 \mathbf{y}^{n}+\mathbf{y}^{n-1} & =\frac{1}{M} \int_{0}^{\Delta t}(\Delta t-\tau)\left(\mathbf{f}^{n}(\tau)+\mathbf{f}^{n}(-\tau)\right) d \tau \\
\mathbf{f}^{n}(\tau) & =-\boldsymbol{\phi}^{n}(\tau)^{*} \mathbf{H}^{\prime}\left(\mathbf{y}^{n}\right) \boldsymbol{\phi}^{n}(\tau) \\
\phi^{n}(\tau) & =\exp \left(-\mathrm{i} \tau \mathbf{H}^{n}\right) \boldsymbol{\psi}^{n}
\end{aligned}
$$

where $\boldsymbol{\psi}^{n}$ can be determined from (19)-(20). Section 5 shows that the scheme (21) can still be implemented efficiently using Lanczos' method, at the expense of computing a number of inner products in addition to (18). The accuracy of this scheme is limited by the recursion for $\boldsymbol{\psi}$.

With $\dot{\mathbf{H}}^{n}=\mathbf{H}^{\prime}\left(\mathbf{y}^{n}\right) \mathbf{v}^{n}$ and $\mathbf{v}^{n}=\left(\mathbf{y}^{n+1}-\mathbf{y}^{n-1}\right) /(2 \Delta t)$, we define the Hermitian matrix

$$
\mathbf{J}^{n}=\int_{0}^{1 / 2} \exp \left(-\mathrm{i} \theta \Delta t \mathbf{H}^{n}\right) \theta \dot{\mathbf{H}}^{n} \exp \left(\mathrm{i} \theta \Delta t \mathbf{H}^{n}\right) d \theta
$$

and consider the following symmetric and norm-preserving scheme:

$$
\begin{aligned}
\boldsymbol{\psi}^{n} & =\exp \left(\mathrm{i} \Delta t^{2} \mathbf{J}^{n}\right) \exp \left(-\mathrm{i} \frac{\Delta t}{2} \mathbf{H}^{n}\right) \boldsymbol{\psi}^{n-1 / 2}, \\
\boldsymbol{\psi}^{n+1 / 2} & =\exp \left(-\mathrm{i} \frac{\Delta t}{2} \mathbf{H}^{n}\right) \exp \left(-\mathrm{i} \Delta t^{2} \overline{\mathbf{J}}^{n}\right) \boldsymbol{\psi}^{n}
\end{aligned}
$$

This formula can be motivated by applying the variation of constants formula to $i \hbar \dot{\boldsymbol{\psi}}=\mathbf{H}^{n} \boldsymbol{\psi}+\left(\mathbf{H}(\mathbf{y})-\mathbf{H}^{n}\right) \boldsymbol{\psi}$. The method can be implemented using Chebyshev approximations to the exponential, see [14].

For the combined scheme (21), (23), second-order error bounds are derived in [14]. These bounds hold independently of the size of the eigenvalues of $\mathbf{T}$, and without assumptions about the smoothness of the solution, which in general is highly oscillatory. 
For the system (16) it is known [5] under non-resonance assumptions that in the limit $m / M \rightarrow 0$ the motion of the classical particle is governed by the Born-Oppenheimer potential (more precisely, by its approximation corresponding to the space discretization (16)),

$$
M \ddot{\mathbf{y}}=-\nabla_{\mathbf{y}} U_{B O}(\mathbf{y})
$$

Letting $m / M \rightarrow 0$ in the numerical method, it can be shown that the solution given by (21) tends to a small perturbation of the Verlet method formally applied to that equation:

$$
\mathbf{y}^{n+1}-2 \mathbf{y}^{n}+\mathbf{y}^{n-1}=\frac{\Delta t^{2}}{M}\left(-\nabla_{\mathbf{y}} U_{B O}\left(\mathbf{y}^{n}\right)+O\left(\Delta t^{2}\right)\right) .
$$

This is apparently not true for (18) and similar schemes with pointwise force evaluation.

Recently, there has been an effort to correct the tensor-product ansatz underlying the QCMD and many other models. In the Configuration Interaction extension of the Classical Separable Potential approach [17], Jungwirth and Gerber use first classical trajectories to guide the selection of important correlation terms and to simplify multidimensional integral evaluations for a subsequent quantum propagation. That approach requires the numerical solution of a large number of 1-dimensional Schrödinger equations with time-dependent Hamiltonian,

$$
i \hbar \dot{\boldsymbol{\psi}}=\mathbf{H}(t) \boldsymbol{\psi}
$$

These can again be solved by the scheme (23), interpreting now $\mathbf{H}^{n}=\mathbf{H}\left(t^{n}\right)$ and $\dot{\mathbf{H}}^{n}=\dot{\mathbf{H}}\left(t^{n}\right)$ in $(23)$ and $(22)$.

\section{Implementation using Lanczos' method}

A common ingredient of all the schemes proposed in this article is the computation of the product of an entire function of a real symmetric matrix with a vector, $\varphi(\mathbf{S}) \mathbf{b}$. In the various schemes, this is needed for $\varphi(z)=\sigma\left(\Delta t^{2} z\right)$, $\phi\left(\Delta t^{2} z\right)$, or $e^{-\mathrm{i} \Delta t z}$, and for $\mathbf{S}=\mathbf{A}$ or $\mathbf{S}=\mathbf{H}$. Computing $\varphi(\mathbf{S}) \mathbf{b}$ is an easy task when the eigendecomposition of $\mathbf{S}$ is available, e. g., when $\mathbf{S}$ is a spectrally discretized Laplacian. However, computing the eigendecomposition of a general symmetric matrix of large dimension is prohibitive because of memory requirements and computational effort, in particular so when a different matrix occurs in every time step.

We here describe the alternative of approximating $\varphi(\mathbf{S}) \mathbf{b}$ via Lanczos' method. The Lanczos process $[18,22]$ recursively generates an orthonormal basis $\mathbf{Q}_{m}=\left[\mathbf{q}_{1}, \ldots, \mathbf{q}_{m}\right]$ of the $m$ th Krylov subspace $\operatorname{span}\left\{\mathbf{b}, \mathbf{S b}, \ldots, \mathbf{S}^{m-1} \mathbf{b}\right\}$ such that

$$
\mathbf{S Q}_{m}=\mathbf{Q}_{m} \mathbf{L}_{m}+\beta_{m}\left[\mathbf{0} \cdots \mathbf{0} \mathbf{q}_{m+1}\right]
$$


with the $m \times m$ tridiagonal Lanczos matrix

$$
\mathbf{L}_{m}=\mathbf{Q}_{m}^{T} \mathbf{S Q}_{m} .
$$

This construction requires one matrix-vector multiplication with $\mathbf{S}$ and two inner products in each recursive step. Therefore, it is not necessary to store $\mathbf{S}$ explicitly as a matrix. The Lanczos process yields the approximation $[21,7,12]$

$$
\varphi(\mathbf{S}) \mathbf{b} \approx \mathbf{Q}_{m} \varphi\left(\mathbf{L}_{m}\right) \mathbf{Q}_{m}^{T} \mathbf{b},
$$

where we note that $\mathbf{Q}_{m}^{T} \mathbf{b}=\|\mathbf{b}\| \cdot\left[\begin{array}{llll}1 & 0 & \cdots & 0\end{array}\right]^{T}$. A robust and inexpensive stopping criterion for the Lanczos iteration based on a generalized residual is described in [15]. Convergence properties are studied in [7,12]. Since $m$ is typically very small compared to the dimension of $\mathbf{S}, \varphi\left(\mathbf{L}_{m}\right)$ can easily be computed by diagonalization of $\mathbf{L}_{m}$,

$$
\mathbf{L}_{m}=\mathbf{U}_{m} \mathbf{D}_{m} \mathbf{U}_{m}^{T}
$$

with an $m \times m$ orthogonal matrix $\mathbf{U}_{m}$ and diagonal matrix $\mathbf{D}_{m}$. This makes the algorithms of Sections 2-4 practical. The required number $m$ of Lanczos steps is at worst of the magnitude of $\left\|\Delta t^{2} \mathbf{A}\right\|$ or $\|\Delta t \mathbf{H}\|$, and often considerably smaller. This may however lead to (relatively mild) time step restrictions.

Finally we discuss the implementation of (21). We approximate (omitting the time superscript $n$ )

$$
\begin{aligned}
\phi(\tau) & =\exp (-\mathrm{i} \tau \mathbf{H}) \boldsymbol{\psi} \\
& \approx \mathbf{Q}_{m} \exp \left(-\mathrm{i} \tau \mathbf{L}_{m}\right) \mathbf{Q}_{m}^{T} \boldsymbol{\psi}=\mathbf{Q}_{m} \mathbf{U}_{m} \exp \left(-\mathrm{i} \tau \mathbf{D}_{m}\right) \mathbf{c}
\end{aligned}
$$

with $\mathbf{c}=\mathbf{U}_{m}^{T} \mathbf{Q}_{m}^{T} \boldsymbol{\psi}$, so that we have

$$
\begin{aligned}
\mathbf{f}(\tau) & =-\boldsymbol{\phi}(\tau)^{*} \mathbf{H}^{\prime} \boldsymbol{\phi}(\tau) \\
& \approx-\mathbf{c}^{*} \exp \left(\mathrm{i} \tau \mathbf{D}_{m}\right) \mathbf{U}_{m}^{T} \mathbf{K}_{m} \mathbf{U}_{m} \exp \left(-\mathrm{i} \tau \mathbf{D}_{m}\right) \mathbf{c} .
\end{aligned}
$$

Here,

$$
\mathbf{K}_{m}=\mathbf{Q}_{m}^{T} \mathbf{H}^{\prime} \mathbf{Q}_{m}
$$

requires the computation of $\mathrm{m}^{2} / 2$ long inner products for each component of $\mathbf{y}$. With this approximation, the integral in (21) is easily computed analytically.

Acknowledgement. We thank Ch. Schütte and P. Nettesheim for stimulating discussions on the topics of this paper.

\section{References}

1. H. C. Andersen. Rattle: A "velocity" version of the Shake algorithm for molecular dynamics calculations. J. Comp. Phys., 52:24-34, 1983.

2. H. J. C. Berendsen and J. Mavri. Quantum simulation of reaction dynamics by density matrix evolution. J. Phys. Chem., 97:13464-13468, 1993. 
3. F. A. Bornemann, P. Nettesheim, and Ch. Schütte. Quantum-classical molecular dynamics as an approximation for full quantum dynamics. J. Chem. Phys., 105(3):1074-1083, 1996.

4. F. A. Bornemann and Ch. Schütte. A mathematical investigation of the CarParrinello method. Preprint SC 96-19, ZIB Berlin, 1996. To appear in Numer. Math.

5. F. A. Bornemann and Ch. Schütte. On the singular limit of the quantumclassical molecular dynamics model. Preprint SC 96-07, ZIB Berlin, 1996. Submitted to SIAM J. Appl. Math.

6. R. Car and M. Parrinello. Unified approach for molecular dynamics and densityfunctional theory. Phys. Rev. Letter, 55:2471-2474, 1985.

7. V. L. Druskin and L. A. Knizhnerman. Krylov subspace approximations of eigenpairs and matrix functions in exact and computer arithmetic. Numer. Lin. Alg. Appl., 2:205-217, 1995.

8. B. García-Archilla, J. M. Sanz-Serna, and R. Skeel. Long-time-step methods for oscillatory differential equations. Applied Mathematics and Computation Reports 1996/7, Universidad de Valladolid, 1996.

9. A. García-Vela, R. B. Gerber, and D. G. Imre. Mixed quantum wave packet/classical trajectory treatment of the photodissociation process $\mathrm{ArHCl}$ $\rightarrow \mathrm{Ar}+\mathrm{H}+\mathrm{Cl}$. J. Chem. Phys., 97:7242-7250, 1992.

10. W. Gautschi. Numerical integration of ordinary differential equations based on trigonometric polynomials. Numer. Math., 3:381-397, 1961.

11. R. B. Gerber, V. Buch, and M. A. Ratner. Time-dependent self-consistent field approximation for intramolecular energy transfer. J. Chem. Phys., 66:30223030, 1982.

12. M. Hochbruck and Ch. Lubich. On Krylov subspace approximations to the matrix exponential operator. SIAM J. Numer. Anal., 34:1911-1925, 1997.

13. M. Hochbruck and Ch. Lubich. A Gautschi-type method for oscillatory secondorder differential equations. Tech. Rep., Universität Tübingen, 1998.

14. M. Hochbruck and Ch. Lubich. Exponential integrators for quantum-classical molecular dynamics. Tech. Rep., Universität Tübingen, 1998. In preparation.

15. M. Hochbruck, Ch. Lubich, and H. Selhofer. Exponential integrators for large systems of differential equations. SIAM J. Sci. Comput., 1998. To appear.

16. J. Hutter, M. E. Tuckerman, and M. Parrinello. Integrating the Car-Parrinello equations. III. Techniques for ultrasoft pseudopotentials. J. Chem. Phys., 102(2):859-871, 1995.

17. P. Jungwirth and R. B. Gerber. Quantum dynamics of large polyatomic systems using a classically based separable potential method. J. Chem. Phys., 102:60466056, 1995.

18. C. Lanczos. Solution of systems of linear equations by minimized iterations. $J$. Res. Nat. Bureau Standards, 49:33-53, 1952.

19. P. Nettesheim, F. A. Bornemann, B. Schmidt, and Ch. Schütte. An explicit and symplectic integrator for quantum-classical molecular dynamics. Chemical Physics Letters, 256:581-588, 1996.

20. P. Nettesheim, Ch. Schütte, M. Hochbruck, and Ch. Lubich. Work in preparation.

21. T. J. Park and J. C. Light. Unitary quantum time evolution by iterative Lanczos reduction. J. Chem. Phys., 85:5870-5876, 1986.

22. B. N. Parlett. The Symmetric Eigenvalue Problem. Prentice-Hall, Englewood Cliffs, N.J., 1980. 
23. J. P. Ryckaert, G. Cicotti, and H. J. Berendsen. Numerical integration of the cartesian equations of motion of a system with constraints: molecular dynamics of $n$-alkanes. J. Comp. Phys., 23:327-341, 1977.

24. M. E. Tuckerman and M. Parrinello. Integrating the Car-Parrinello equations. I. Basic integration techniques. J. Chem. Phys., 101(2):1302-1315, 1994.

25. M. E. Tuckerman and M. Parrinello. Integrating the Car-Parrinello equations. II. Multiple time scale techniques. J. Chem. Phys., 101(2):1316-1329, 1994. 